\title{
On mesh sensitive calculations in 3D finite element modeling of electric machines
}

\author{
Robert Kaczmarek $^{1}$, Pau Masgrau ${ }^{2}$ and Mehdi Saheli ${ }^{3}$ \\ ${ }^{1}$ Department of Power \&EnergySystems \\ SUPELEC, Ecole Supérieure d'Électricité \\ 3 rue Joliot Curie, 91192 Gif-sur-Yvette, France \\ Phone +33169851532,robert.kaczmarek@ supelec.fr, \\ ${ }^{2}$ was with Supélec in this project, now with POLYLUX S.L. \\ C/ Boters, 3B - 08290Cerdanyola del Vallès, Spain.pau.masgrau@gmail.com \\ ${ }^{3}$ RENAULT / DELT'A/ Service Conception \& Ingénierie numérique \\ 1 avenue du Golf 78288 Guyancourt, France.mehdi.saheli@ renault.com
}

\begin{abstract}
The study has been carried on for mesh sensitivity in 3D finite element (FEM) calculation of magnetic losses in laminations of an electric machine. Looking for stabilization of results with growing refinement of meshing, we analyse the sensitivity-free ranges of mesh density for several uncorrelated pattern sets of the machine under study. The mesh sensitivity is lesser for lower frequency. Together with algorithmical limitation of the FEM calculation of magnetic losses, actually leading only to classical losses, the mesh sensitivity contests this calculation method even at high frequency. As for the FEM field density calculation, it can be locally relevant provided that a time and power consuming research determines the sensitivity-free mesh density.
\end{abstract}

Key words-Finite element method, electrical machine design, eddy current losses.

\section{Introduction}

Seven decades after introduction of the finite element calculation methods [1] the mesh sensitivity is still a major methodological problem in industrial applications. In simple structures efficient mathematical instruments of local evaluation of the mesh pattern induced error are reported [2]. Such error estimators confirm the sensibility of calculated results on meshing.

As for the global control of the mesh sensibility, different procedures are recently discussed in computational physics, still for relatively simple structures, as is the case [3] with the moving mesh methods for solving phase-field equations. Here the structural simplicity allow for relevance of heavy algorithmical involvement. Another type of incentive to examine the divergence induced by mesh refining is with FEM applications in mechanics [4-7] where maximum values of stress is used as a failure criterion.

On the contrary, the maximum field value may appear as not necessarily critical parameter in electromagnetical applications with magnetic saturation code limiting the eventually divergent calculation. This circumstance, together with structural complexity of magnetic circuits in power applications can be among the reasons of a relative inattention to mesh sensitivity here. When a relevant analysis happens in papers on FEM applications in electric machines, what is rather unusual, then mainly in simple 2D structures with help of sophisticated procedures.

In this area Lowrie, Lukin and Shumlak[8] present a 2D study on mesh deformations to evaluate their impact on the error. A high-order finite element code is used to study effects of various mesh distortions on quality of solution. It is observed that increasing the level of mesh deformation enhances the error magnitude. The error sensitivity for each distortion degree depends on the type of distortion. In conclusion the authors advise cautiousness about how the mesh might influence their particular problem.

Hallero and Rylander[9] consider 2D electromagnetic problems of both electric and magnetic losses in a circular metal cylinder. The combination of finite difference time domain (FDTD) scheme applied to large homogeneous regions and of the finite-element method (FEM) for regions with complicated geometry is attractive for many scattering problems. The homogeneous regions are divided in rectangular Cartesian structured mesh whereas zones of complicated geometries are given an unstructured mesh set of triangles in the vicinity of curved boundaries. At the interface that connects the structured and unstructured sets each edge is shared by one rectangle and one triangle. The results show that the relative error of the solution decreases when the mesh resolution rises.

The relevance of the mesh induced uncertainty somewhat contradicts opinion[10, 11], that the 2D nonlinear electromechanical alternating fields FEM analysis in time and frequency domains is already mature and industrially accepted. It may be true in simple structures. 
In FEM industrial applications some practical meshrefinement approaches may be useful, like making more subdivisions over stator winding bars' height and width when looking for eddy currents in these bars [12]. In few cases when such approaches are reported, the papers point to negligible or rapidly disappearing mesh sensitivity: in the cited 2D example [12] the comparison for three mesh compositions concludes on rapid stabilization of results. Similar insensitivity is presented in comparison of results on five different mesh patterns[13] in thermo-electrical FEM 3D modeling of a DC motor; when doubling the number of mesh cells, the variation of calculated flux rate (actually the heat transfer) was only of $3 \%$.

The assessment of the weak sensibility corresponds to a kind of intuitive confidence in numerical results produced by a given FEM model, but is in contradiction with warnings $[14,15]$ on importance of the phenomenon. We think it should always be tested by refining the actual mesh pattern.

Usual meshing patterns in electrical machines show geometrical elements dimensioned e.g. in the range from sub-centimetre size in peripheral parts of the armature to sub-millimetre size in the gap area. The complexity of machine structure makes irrelevant both adaptive element methods and direct verification of results, as the analytical search for continuous solution in machines can only be very approximate. Actually, we start with designing of mesh profile in sensible areas, e.g. teeth or gap, using some experience-based rules like avoiding important differences in mesh dimensions, particularly in adjacent elements. Then the meshing goes on with semi-automatic discretization.

Such a procedure may be relevant only if we get stabilization of results with growing refinement of meshing. This stabilization should be repetitive for several, at least two different pattern sets. The research for sensibility-free meshing is relevant with linearly modelled machine.

This is the procedure we have applied in modelling an electric traction motor of several tens of $\mathrm{KW}$.

\section{Methodology of calculation}

Usually the finite element calculation of magnetic losses operates out of induced currents, these currents being result of double derivation leading from vector potential $A$ to field density $B$ and from $B$ to its curl. Actually the $\partial A / \partial x$ derivation induces sensitivity on meshing because finite discretization steps over time and space result in discontinuity across mesh borders of the derivatives of continuous functions. The FEM solution tends toward the continuous one (i.e. toward the actual field value) when the mesh dimensions and the calculation steps tend toward zero.

This kind of digitally induced sensitivity is a factor of imprecision linked to mesh dimensions and with calculation step, possibly important in the field calculation, and even enhanced in calculation of induced currents, where multiple derivations of field is necessary. We assume the calculation step being sufficiently small and we go on with analysis of sensitivity on the sole meshing.

All the calculations have been brought about with the Ansys mechanical package which is an ancient module of Ansys for electromagnetical analysis. Ansys calculates the dissipated heat for each element.

Two Ansys modules have been used for calculation: the time module and the harmonic module. The time module gives time related scalar values of dissipated heat for each of time steps over the period of interest, whereas the harmonic analysis gives time-averaged values over one period, these values being stored in "Real" and "Imaginary" data sets. Then a post-treatment in Matlab gives losses for all the elements averaged over one period.

\section{Modelling machine}

One pair of poles is modelled taking advantage of periodic conditions.Initially the model was composed by 3 laminations of magnetic material and 1 of air, and later on we have transformed the 3 laminations into one for ergonomical reasons. The rotor is geometrically immobile with reconfiguration of node coupling in the air gap creating the rotor's virtual movement.

Periodic conditions and virtual movement are set by coupling a group of nodes. It is done by forcing the assignation of the same DOF values to the coupled nodes, meaning a magnetic contact between these nodes. Coupled nodes belonging to steel elements share magnetic potential vector $\left(\mathrm{A}_{\mathrm{x}}, \mathrm{A}_{\mathrm{y}}, \mathrm{A}_{\mathrm{z}}\right)$ and Voltage (VOLT) values as A-VOLT elements, and the coupled nodes belonging to air share magnetic potential vector (Ax, Ay, Az) values as A elements.

The machine is modelled as magnetically and electrically linear. Stator and rotor electrical steel are modelled as constant resistivity conductors. Its permeability takes different values depending on the magnetic flux density $\boldsymbol{B}$ amplitude and frequency. Induced currents will be calculated with help of the vector potential $\boldsymbol{A}$ computed in three axis directions and of the scalar potential VOLT. It means the degrees of liberty (DOF) of these elements will be $A_{X}, A_{Y}, A_{Z}$ and VOLT. The VOLT parameter has one stator node and one rotor node assigned as references.

The rest of the model is characterized by non conductor conditions and $\mu_{\mathrm{r}}=1$. The DOF for the elements located in these areas is the vector potential A. The current density (stator supply) is imposed in the stator slots.

The basic equations used for Ansys for these conductor areas are summarized in the table 1: 
Table I. -Parameters and physics laws applied to Ansys materials in Renault II model

\begin{tabular}{|c|c|c|c|}
\cline { 2 - 4 } \multicolumn{1}{c|}{} & $\begin{array}{c}\text { Stator and rotor } \\
\text { electrical steel }\end{array}$ & Stator slots & $\begin{array}{c}\text { Air gap } / \\
\text { Air }\end{array}$ \\
\hline $\begin{array}{c}\text { Param } \\
\text { eter }\end{array}$ & $\begin{array}{c}\rho=37.6 \mathrm{e}^{-8} \Omega \cdot \mathrm{m} \\
\mu_{\mathrm{r}}\end{array}$ & $\mu_{\mathrm{r}}=1$ & $\mu_{\mathrm{r}}=1$ \\
\hline $\begin{array}{c}\text { Elem. } \\
\text { type }\end{array}$ & $\begin{array}{c}\text { SOLID97 } \\
\text { A-VOLT } »\end{array}$ & $\begin{array}{c}\text { SOLID97 } \\
\text { A } »\end{array}$ & $\begin{array}{c}\text { SOLID97 } \\
\text { A » }\end{array}$ \\
\hline DOF & $\mathrm{AX}, \mathrm{AY}, \mathrm{AZ}, \mathrm{VOLT}$ & $\begin{array}{c}\mathrm{AX}, \mathrm{AY}, \\
\mathrm{AZ}\end{array}$ & $\begin{array}{c}\mathrm{AX}, \mathrm{AY}, \\
\mathrm{AZ}\end{array}$ \\
\hline Laws & $\nabla \cdot\left(-\sigma \frac{\partial A}{\partial t}-\sigma \nabla V\right)=0$ & $\begin{array}{c}\nabla \times H=J_{s} \\
\mathrm{~s}: \mathrm{source}\end{array}$ & $\nabla \times H=0$ \\
& $P_{k}=\rho \cdot j_{k}^{2}$ & & \\
\hline
\end{tabular}

\section{Mesh-pattern sensible results}

\section{A. Tests on local parameter: flux induction $B$}

The FE model has been checked in the Ansys temporary calculation mode ("transient analysis") under the same operating conditions: with simulation curves corresponding to two electrical periods and the maximal value taken in the second period.

At first, 4 different mesh patterns have been tested with 57000, 64000, 79000 and finally 107000 elements pattern. Starting from the $57 \mathrm{k}$ mesh pattern, the $64 \mathrm{k}$ and the $107 \mathrm{k}$ mesh pattern have been developed by enhancing the steel meshing density. On the other hand the $79 \mathrm{k}$ mesh pattern has been designed by raising the number of the air elements out of the $64 \mathrm{k}$ pattern. Several points of control have been fixed at key locations of the model to ensure the adequate comparison of the $\mathrm{B}$ parameter values.

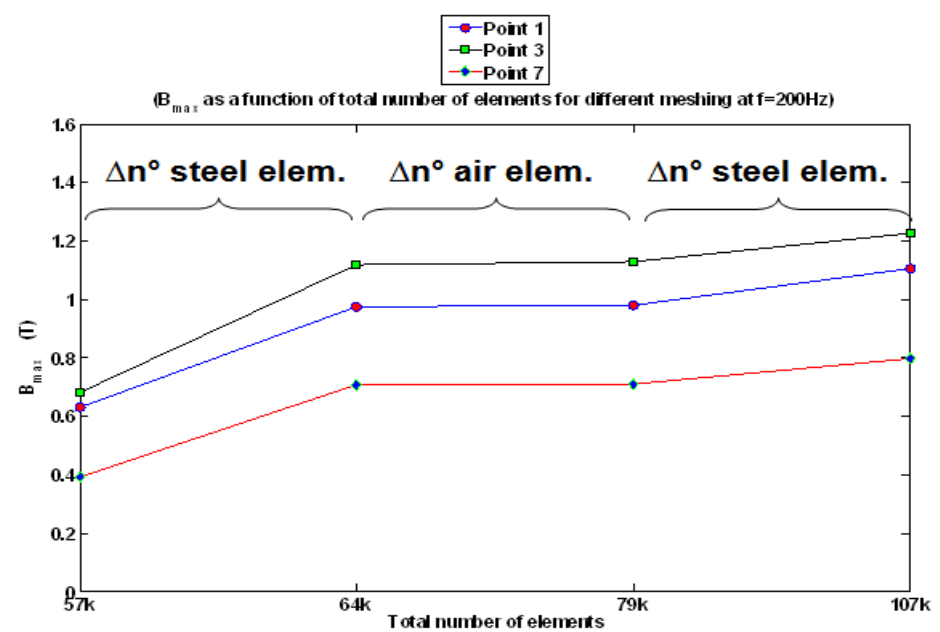

Fig. 4. $\mathrm{B}_{\max }$ for 4 different mesh patterns $(57 \mathrm{k}, 64 \mathrm{k}, 79 \mathrm{k}, 107 \mathrm{k})$ in three points: armature outermost side point 1 , armature innermost side point 2 , and in the center of stator tooth point 3
The results presented on Fig. 4 show the $B$ values rising significantly when the steel mesh is refined, but keeping constant when the mesh is refined only in the air areas. As a matter of fact, the field values on the $79 \mathrm{k}$ mesh pattern are identical as the $64 \mathrm{k}$, both differing only by the air element density.

\section{B. Test on global parameter: eddy current losses}

However, the local parameter B is quite an imprecise tool of analysis when the mesh patterns get refined because the comparison takes place not exactly at the same location, even though the error may be considered as irrelevant in our test conditions. More important drawback of testing local B on mesh pattern sensitivity results from the very locality of the parameter. The truly relevant test should be carried on in respect to some global parameter. Such a global parameter is the machine's eddy current losses.

The tests have been carried on two series of mesh patterns, from $12 \mathrm{k}$ to $140 \mathrm{kstator}$ elements, in which the stator eddy current losses are analyzed.

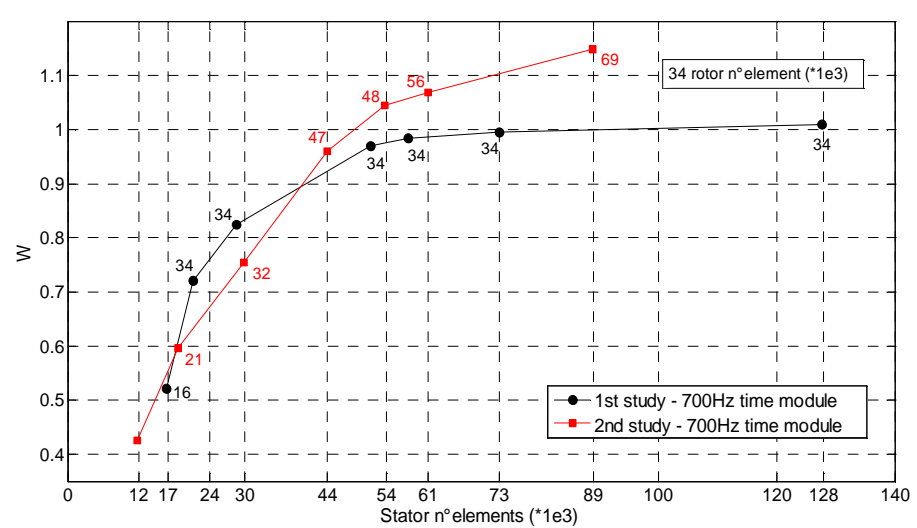

Fig. 5. Stator eddy current losses calculated by transient module at $700 \mathrm{~Hz}$. The numbers show the number of elements (in thousands) in the rotor

The results show again important sensitivity on meshing (Fig. 5), the stator losses rising with refinement of meshing and the curve of sensitivity showing asymptotical tendency. The mesh density range starting at about 60000 elements appears as sensitivity-free for two uncorrelated meshing sets. Both stator and rotor mesh density contribute to reach the asymptotical zone of the calculated value of stator eddy current losses.

In order to minimize the computational stress some simple rules can be applied, like diminishing of number of radial layers in lamination model (Fig. 6).
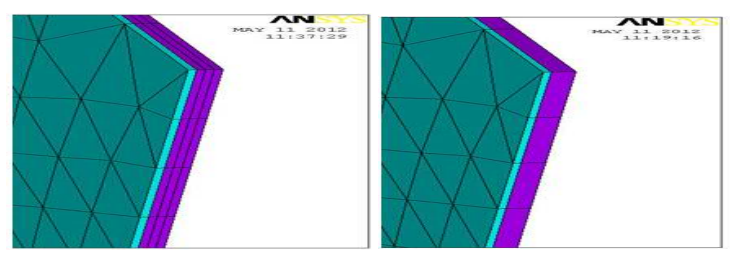

Fig. 6. Air and steel laminations for 3-layer (left) and 1-layer modelling 
The sensibility on meshing is checked on both models. The asymptotic value of losses calculated in one layer lamination is obtained for much lower number of meshes compared to the full three-layer model (Fig. 7)

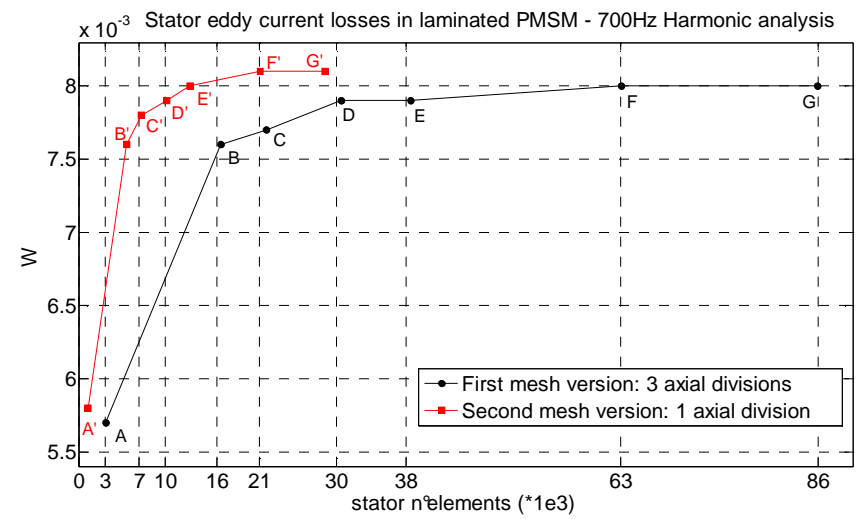

Fig. 7.Sensitivity test onsteellaminationsfor 3-layer (lower curve) and 1-layer steellamination

\section{Frequency study}

The FEM simulation being based on digital calculations of derivatives of the type $\partial A / \partial x$ we can assume both frequency and meshing refinement as factors of sensitivity. This was confirmed (Fig. 8) in a series of 7 different mesh patterns at two different frequencies $(267 \mathrm{~Hz}$ and $700 \mathrm{~Hz})$.

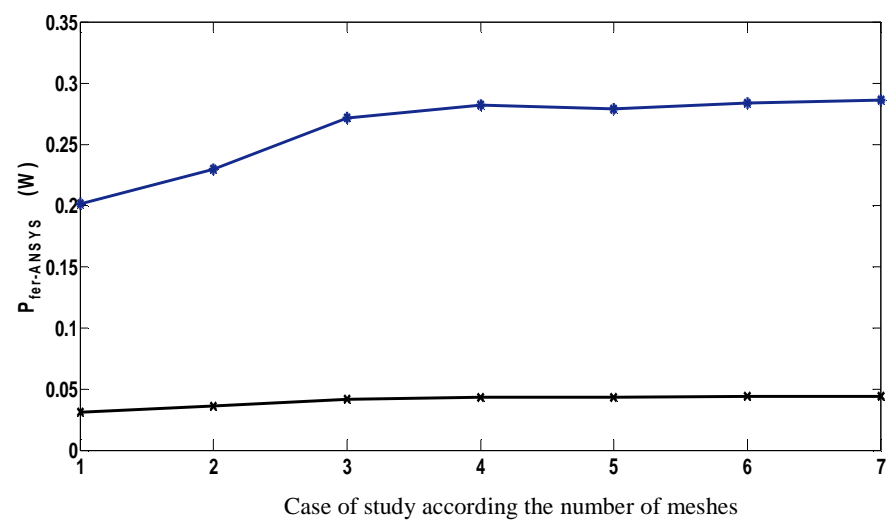

Fig. 8.Stator losses at different frequencies for different mesh patterns for $700 \mathrm{~Hz}$ (above) and $267 \mathrm{~Hz}$.

At $267 \mathrm{~Hz}$ the mesh sensitivity is lesser with apparent increment of losses of $30 \%$ compared to $40 \%$ at $700 \mathrm{~Hz}$.

\section{Conclusion}

The study has shown importance of sensitivity on meshing of FEM field and losses calculations. Growing with frequency, the phenomenon can be more easily neglected at $50 \mathrm{~Hz}$. Stability of results should always be tested and the mesh pattern suitable for calculation should be determined by densification of meshing previously to field values calculation. This turns the procedure extremely time and memory consuming.

The variety of situation in complex structures' modelling, together with fundamental limitation of the FEM losses calculation, where all we can do are classical losses, makes us sceptical as to usefulness of this technique for current oriented calculation of magnetic losses. The B-oriented losses formulae would be exempt of these drawbacks, provided the data on local B values are available.

\section{References}

[1] H.WangHrennikoff, A. Solution of problems in elasticity by the framework method, J. Appl. Mech., A8, 169-175, (1941)

[2] 'La maitrise de l'erreur due à la discrétisation par éléments finis » after P.Ladevèze and J.-P.Pelle"Mastering Calculations in Linear and Nonlinear Mechanics", Springer Verl. Mech .Engng. Ser.

[3] H.Wang and R.Li "Mesh sensitivity for numerical solutions of phase-field equations using r-adaptive finite element methods" Commun. Comp. Phys. 1 (1) pp. 1-19 (2006)

[4] C. Basaran and Y. Zhao "Mesh sensitivity and FEA for multi-layered electronic packaging" J. Electron Packaging 123 (3) 2001

[5] A.Munjiza and N.W.M.John "Mesh size sensitivity of the combined FEM/DEM fracture and fragmentation algorithms" Engineering Fracture Mechanics, 69 (2) pp. 281-295 (2002)

[6] J.Cervenka, Z.Bazant and M.Wierer "Equivalent localization element for crack band approach to meshsensitivity in microplane model" Int. J. Numer. Meth. Engng62 pp. 700-726 (2005)

[7] S Badgujar, H Vaghela, N Shah, R Bhattacharya and B Sarkar "mesh sensitivity study and optimization of fixed support for ITER torus and cryostat cryoline"J. Phys.: Conf. Ser.208 012010

[8] W. Lowrie, V.S. Lukin, U. Shumlak. A priori mesh quality metric error analysis applied to a high-order finite element method, J. Comp. Phys.230 (14), 5564 (2011)

[9] Tomas Hallerod, Thomas Rylander. Electric and magnetic losses modeled by a stable hybrid with explicit-implicit time-stepping for Maxwell's equations, Journal of Computational Physics 227 (9), pp. 4499-4511 (2007)

[10] Siu-Lau Ho "Finite element analysis of machines" in "Electrical Engineering" vol. III , edited by Department of Electrical Engineering, The Hong Kong Polytechnic University, Hong Kong,

[11] D.Ladas et al « An energy based approach of electromagnetism applied to adaptive meshing and error criteria" IEEE Trans. Magn. 44 (6) pp 1246 - 1249 (2008)

[12] M.J.Islam «Finite-element analysis of Eddy currents in the form-wound multi-conductor windings of electrical machines" Doctoral dissertation at Helsinki University of

[13] R. Amanatidou «CFD measurements of the cooling air in a DC motor", doctoral dissertation atMalardalen University pp. 49 - 52 (2008)

[14] S. Dufour, G. Vinsard and B. Laporte "2-D Adaptive Mesh with movement" IEEE Trans. Magn., 37(5), pp. 3482$3485(2001)$

[15] Besson, P.Nikkola, M.Jaccard « Agir sur le bobinage pour reduire les pertes au rotor »electrosuisse bulletin 6 pp. 28 $33(2011)$ 